\title{
Eye plaque brachytherapy versus enucleation for ocular melanoma: an analysis from the National Cancer Database
}

\author{
Jay A. Messer, MD',2, Raed J. Zuhour, MD1,2, Wagar Haque, MD², Gary D. Lewis, MD³, Amy C. Schefler, MD4, \\ Andrew Wong, MD',2, Eric H. Bernicker, MD5, Patricia Chevez-Barrios, MD6. Enzhuo Michelle Quan, PhD², \\ Andrew Farach, MD², E. Brian Butler, MD², Sandra S. Hatch, MD',7, Bin S. Teh, MD² \\ 'Department of Radiation Oncology, The University of Texas Medical Branch, Galveston, TX, USA, ${ }^{2}$ Department of Radiation Oncology. \\ Houston Methodist Hospital, Houston, TX, USA, ${ }^{3}$ Department of Radiation Oncology, University of Arkansas for Medical Sciences, Little Rock, \\ AR, USA, ${ }^{4}$ Department of Ophthalmology, Houston Methodist Hospital, Houston, TX, USA, ${ }^{5}$ Department of Medical Oncology, Houston \\ Methodist Hospital, Houston, TX, USA, 'Department of Pathology, Houston Methodist Hospital, Houston, TX, USA, 7 Department of Radiation \\ Oncology, MD Anderson Cancer Center, Houston, TX, USA
}

\begin{abstract}
Purpose: There is no current randomized data comparing the efficacy of brachytherapy and enucleation for patients with larger sized tumors. The purpose of the present study was to use a large, contemporary database to determine current practice patterns and compare survival outcomes between different management options for patients with choroidal melanoma of various sizes.

Material and methods: The National Cancer Database was queried (2004-2014) for histologically-confirmed choroidal melanoma for patients treated with brachytherapy versus enucleation. Chi-square test was used to compare categorized demographic and clinical variables in both arms. Kaplan-Meier analysis evaluated overall survival (OS). Cox proportional hazards assessment determined variables associated with OS. Patients were divided into cohorts representing small, medium, and large tumors. Propensity scores matching (PSM) was utilized to compare more similar cohorts.

Results: A total of 7,096 patients met the selection criteria; 5,501 (78\%) patients received brachytherapy and 1,595 (22\%) patients were treated with enucleation. After PSM, 5-yr OS for small tumors was $87 \%$ vs. $64 \%$, for medium tumors was $77 \%$ vs. $57 \%$, and for large tumors was $68 \%$ vs. $46 \%$ for brachytherapy and enucleation, respectively $(p<0.001)$. Following PSM, multivariate Cox regression found older age (hazard ratio $[\mathrm{HR}]=1.76,95 \%$ confidence interval $[\mathrm{CI}]=1.51-2.06)$, more comorbidities $(\mathrm{HR}=1.46,95 \% \mathrm{CI}=1.25-1.70)$, extraocular extension $(\mathrm{EOE})(\mathrm{HR}=1.25$, $95 \% \mathrm{CI}=1.06-1.48)$, ciliary body invasion $(\mathrm{CBI})(\mathrm{HR}=1.20,95 \% \mathrm{CI}=1.02-1.40)$, and larger size $(\mathrm{HR}=1.52,95 \%$ $\mathrm{CI}=1.40-1.66)$ were negative prognosticators of survival. Brachytherapy was a positive prognosticator of survival $(\mathrm{HR}=0.45,95 \% \mathrm{CI}=0.40-0.51)$.

Conclusions: Patients selected for brachytherapy had improved survival compared to enucleation in all size cohorts. EOE and CBI are significantly higher in the enucleation cohort and are important negative prognosticators for survival selected against patients having brachytherapy. Brachytherapy is a reasonable treatment option for certain patients with large size tumors.

J Contemp Brachytherapy 2020; 12, 4: 303-310 DOl: https://doi.org/10.5114/jcb.2020.98108
\end{abstract}

Key words: ocular melanoma, eye plaque brachytherapy, enucleation, NCDB, National Cancer Database, large tumors.

\section{Purpose}

Ocular melanoma (OM) accounts for $2.9 \%$ of all melanomas in the United States and is the most common primary intraocular malignancy in adults [1]. Approximate$1 y, 95 \%$ of OM arise from uveal melanocytes, including iris, ciliary body, and choroid. The majority arise from the choroid and ciliary body (90-95\%), which are more likely to be fatal than tumors in the iris [2]. As such, tumors of the choroid and ciliary body are treated more aggressively and have been treated historically with enucleation.

In recent decades, studies have been focusing on adopting treatments with the potential for globe perseveration without compromising survival. Among those
Address for correspondence: Bin S. Teh, MD, FACR, FASTRO, FACRO, Prof., Vice Chair, and Full Member, Department of Radiation Oncology, Houston Methodist Hospital, Cancer Center, and Research Institute, Weill Cornell Medical College, 6565 Fannin, Ste\#DB1-077, Houston, Texas 77030, USA, phone: +1 713-441-4800, fax: +1 713-441-4493, e-mail: bteh@houstonmethodist.org
Received: 11.12.2019

Accepted: 10.05 .2020

Published: 21.08.2020 
treatments is eye plaque brachytherapy (EPBT), where a plaque with radioactive sources is surgically implanted on the sclera overlying the tumor and subsequently removed, following dose delivery. The Collaborative Ocular Melanoma Study (COMS) group conducted a phase III randomized trial that demonstrated medical equipoise between patients with medium sized OM treated with either enucleation or EPBT, with no survival differences found between the treatment cohorts [3]. Overall survival was about $81 \%$ at 5 years and $57 \%$ at 12 years in both arms [4]. The inclusion criteria were defined as medium sized tumors, with height between 2.5-10 mm and diameter less than or equal to $16 \mathrm{~mm}$. This study established eye plaque brachytherapy as a standard of care for patients with medium sized choroidal melanoma tumors.

The National Comprehensive Cancer Network (NCCN) recommendations include particle beam radiotherapy, stereotactic radiosurgery (SRS), or enucleation for large sized tumors (diameter $>18 \mathrm{~mm}$, height $>10 \mathrm{~mm}$ ) [5]. EPBT is not listed as a treatment option for large size tumors, but it is for small size tumors (diameter $<18 \mathrm{~mm}$, height $<2.5 \mathrm{~mm}$ ) and medium (diameter $<18 \mathrm{~mm}$, height 2.5-10 mm). The American Brachytherapy Society (ABS) and the American Joint Committee on Cancer (AJCC) guidelines recommendations state T1, T2, T3, and T4a-d uveal melanoma patients can be treated, after counseling about likely vision, eye retention, and local control outcomes. The ABS guidelines list contraindications to EPBT include large size tumors with extraocular extension (AJCC T4e), basal diameters that exceed the limits of brachytherapy, blind painful eyes, and those with no light perception vision [6]. Retrospective and prospective cohort studies have demonstrated acceptable local control, with $87-94 \%$ for large sized OM tumors treated with EPBT [7,8].

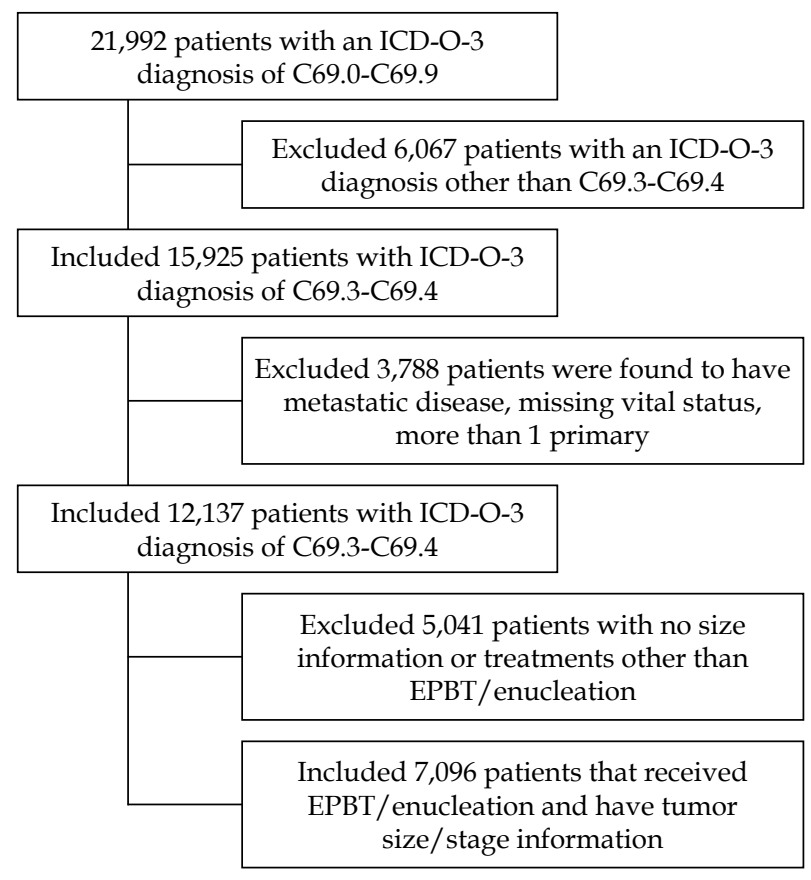

Fig. 1. Consort diagram

EPBT-eye plaque brachytherapy, C69.3-malignant neoplasm of choroid, C69.4 - malignant neoplasm of ciliary body
There is no current randomized data comparing the efficacy of brachytherapy and enucleation for patients with large tumors. Based on current improvements in technology for delivery of EPBT, we hypothesized that the use of EPBT would result in equivalent survival outcomes for larger sized tumors, which was the impetus for the current study [9]. The purpose of the present study was to investigate current practice patterns and compare survival outcomes between different treatment options for patients with choroidal melanoma of all sizes using a large, contemporary database.

\section{Material and methods}

The National Cancer Database (NCDB) is a national hospital-based cancer registry that is co-sponsored by the American College of Surgeons (ACoS) and the American Cancer Society. The NCDB collects data from more than 1,500 hospitals with ACoS-accredited cancer programs, accounting for $70 \%$ of all newly diagnosed cancers in the United States $[10,11,12,13]$. The most recent data from the NCDB included records from 2004 to 2014. Institutional review board approval was not required for this study, as only de-identified information is stored in the database.

For this study, the NCDB was queried for histologically-confirmed choroidal or ciliary body melanoma for patients $\geq 18$ years old. Inclusion criteria consisted of patients with N0 disease, M0 disease, those who have OM as their first and only malignancy, and those who have a known vital status (Figure 1). Patients were divided into two cohorts: those treated with brachytherapy alone versus enucleation alone. While EPBT is commonly used, the NCDB does not specifically mention the exact type of brachytherapy device. Demographic and clinical data included age, sex, treatment facility type, treatment year, race, insurance status, Charlson/Deyo comorbidities score, distance to treatment, time to starting radiation, and medium income of the patient's home zip code were included [14]. The Charlson/Deyo comorbidities was applied, since it is the only such score used by the NCDB. Some patients were missing either staging information or tumor dimension measurements. In order to minimize biases introduced by complete case analyses, patients with tumor dimension information only or those who have tumor dimension information and staged using the American Joint Committee on Cancer (AJCC) $7^{\text {th }}$ edition, were restaged using the AJCC $6^{\text {th }}$ edition. This approach was employed as this staging scheme more closely resembled size criteria used in the COMS trials and NCCN that allows for the inclusion of largest proportion of patients. Patients were divided into cohorts representing small, medium, and large tumors (Table 1). For patients that had both tumor size and staging information, the highest level was used. Ciliary body invasion (CBI) or extraocular extension (EOE) were also evaluated.

All statistical tests were two-sided, with a threshold $p$-value lower than 0.05 for statistical significance, and were performed using SPSS Statistics, version 23 (IBM Corporation, Armonk, NY, USA). The $\chi^{2}$ test were used to compare categorized demographic and clinical variables in both arms. The Kaplan-Meier method was ap- 
Table 1. Size definitions comparing COMS size and the AJCC $6^{\text {th }}$ edition

\begin{tabular}{lccccc} 
Study size criteria & \multicolumn{2}{c}{ AJCC $6^{\text {th }}$ edition (used for this study) } & \multicolumn{2}{c}{ COMS size (reference) } \\
\cline { 2 - 6 } & Stage & Apical height & Basal diameter & Apical height & Basal diameter \\
\hline Small & T1 & $\leq 2.5 \mathrm{~mm}$ & $\leq 10 \mathrm{~mm}$ & $1.5-2.4 \mathrm{~mm}$ & $5-16 \mathrm{~mm}$ \\
\hline Medium & T2 & $>2.5-10 \mathrm{~mm}$ & $>10-16 \mathrm{~mm}$ & $2.5-10 \mathrm{~mm}$ & $\leq 16 \mathrm{~mm}$ \\
\hline Large & T3-T4 & $>10 \mathrm{~mm}$ & $>16 \mathrm{~mm}$ & $>10 \mathrm{~mm}$ & $>16 \mathrm{~mm}$
\end{tabular}

plied for survival analysis, and comparisons between the two treatment paradigms were performed with the log-rank test for all patients. Overall survival (OS) was defined as the interval between the date of diagnosis and the date of death from any cause or last contact. OS is the only disease-related outcome listed in the NCDB. Multivariable Cox proportional hazards modeling was additionally employed to identify variables associated with OS in the entire cohort. Propensity score matching (PSM) was conducted, which used propensity scores based on differences in demographic and clinical variables (age, comorbidity score, treatment distance, facility, education level, insurance, income level, sex, race, city, tumor size, and extraocular extension), and a $1: 1$ matched cohort of brachytherapy and enucleation patients were created using the MatchIt function in R, version 3.5.2 (the R Foundation for Statistical Computing, 2015) [15]. The matched cohorts of 3,190 patients (1,595 in each group) were separately analyzed with the Kaplan-Meier and Cox proportional hazards analyses in the fashion described above.

\section{Results}

A total of 7,096 patients met the inclusion criteria, approximately $35 \%$ of patients treated in the USA in this time period; $5,501(78 \%)$ patients received brachytherapy and 1,595 (22\%) of patients were treated with enucleation (Figure 1) [16]. Median follow-up was 55 and 38 months for brachytherapy and enucleation, respectively. Brachytherapy patients were more likely to be from educated zip codes, have private insurance, live farther away from where they were treated, and have a higher Charlson/Deyo comorbidities score (Table 2). Enucleation patients were more likely to be from low-income zip

Table 2. Demographic data comparison for all patients

\begin{tabular}{|c|c|c|c|c|c|}
\hline Characteristic & Brachytherapy & $\%$ & Enucleation & $\%$ & $p$-value \\
\hline Age ( $\geq 60$ years) & 3,069 & 56 & 871 & 55 & 0.403 \\
\hline Sex (male) & 2,764 & 50 & 910 & 57 & $<0.001^{*}$ \\
\hline Diagnosis after 2010 & 2,296 & 42 & 645 & 40 & 0.354 \\
\hline Academic center & 3,621 & 66 & 1,076 & 68 & 0.224 \\
\hline White race & 5,298 & 96 & 1,503 & 94 & $<0.001^{*}$ \\
\hline Uninsured & 160 & 3 & 113 & 7 & $<0.001^{*}$ \\
\hline Private & 2,944 & 54 & 709 & 45 & $<0.001^{*}$ \\
\hline Government & 2,310 & 42 & 716 & 45 & $0.039^{*}$ \\
\hline High school dropout > 21\% & 702 & 13 & 235 & 15 & $0.041^{*}$ \\
\hline Median income $<\$ 38,000$ & 861 & 16 & 283 & 18 & $0.046^{*}$ \\
\hline Population $<250,000$ & 1,903 & 35 & 592 & 37 & 0.063 \\
\hline Distance to treatment $>60 \mathrm{mi}$ & 2,867 & 52 & 691 & 43 & $<0.001^{*}$ \\
\hline Charlson/Deyo score $\geq 1$ & 938 & 17 & 221 & 14 & $0.002^{*}$ \\
\hline Extraocular extension & 171 & 3 & 237 & 15 & $<0.001^{*}$ \\
\hline Ciliary body & 388 & 7 & 243 & 15 & $<0.001^{*}$ \\
\hline cOMS small & 1,183 & 22 & 331 & 21 & 0.518 \\
\hline COMS medium & 3,641 & 66 & 612 & 38 & $<0.001^{*}$ \\
\hline COMS large & 677 & 12 & 652 & 41 & $<0.001^{*}$ \\
\hline Total & 5,501 & 78 & 1,595 & 22 & 7,096 \\
\hline
\end{tabular}

*Denotes significance 
Table 3. 5-year overall survival (OS) for all patients

\begin{tabular}{lccccc} 
Treatment & $n$ patients & Mean follow-up (months) & Median follow-up (months) & Range & 5-year OS \\
\hline Brachytherapy & 5,501 & 61 & 55 & $0-156$ & $80.0 \%$ \\
\hline Enucleation & 1,595 & 48 & 38 & $0-153$ & $54.0 \%$ \\
\hline Total & 7,096 & - & - & - & $p<0.001$
\end{tabular}

Table 4. 5-year overall survival (OS) for patients divided by COMS size for all patients

\begin{tabular}{|c|c|c|c|c|c|}
\hline \multirow[t]{2}{*}{ COMS size } & \multicolumn{2}{|c|}{ Brachytherapy cohort } & \multicolumn{2}{|c|}{ Enucleation cohort } & \multirow[t]{2}{*}{ Log rank } \\
\hline & $n$ patients & 5-year OS & $n$ patients & 5-year OS & \\
\hline Small & 1,183 & $86.4 \%$ & 331 & $64.1 \%$ & $p<0.001$ \\
\hline Medium & 3,641 & $80.2 \%$ & 612 & $56.6 \%$ & $p<0.001$ \\
\hline Large & 677 & $67.3 \%$ & 652 & $45.7 \%$ & $p<0.001$ \\
\hline
\end{tabular}

codes, have government insurance, be uninsured, have $\mathrm{EOE}$, have CBI, and have large size tumors (Table 2).

Amongst all patients, 5-year overall survival (5-yr OS) was $80 \%$ vs. $54 \%$ for brachytherapy and enucleation, respectively $(p<0.001)$ (Table 3). 5-yr OS for small tumors was $86 \%$ vs. $64 \%$, for medium tumors was $80 \%$ vs. $57 \%$, and for large tumors was $67 \%$ vs. $46 \%$ for brachytherapy and enucleation, respectively $(p<0.001)$ (Table 4) Kaplan-Meier curves show survival trend for all patients divided into size cohorts (Figure 2A).

After propensity matching, the treatment cohorts had no difference in demographic/clinical variables, except for more EOE in the enucleation cohort (15\% vs. $10 \%, p<0.001$ ) (Table 5). 5 -yr OS was $76 \%$ vs. $54 \%$ for brachytherapy and enucleation, respectively $(p<0.00)$ (Table 6). 5-yr OS for small tumors was $87 \%$ vs. $64 \%$, for medium tumors was $77 \%$ vs. $57 \%$, and for large tumors was $68 \%$ vs. $46 \%$ for brachytherapy and enucleation, respectively $(p<0.001)$ (Table 7$)$. Kaplan-Meier curves show survival trend after PSM divided into size cohorts (Figure 2B).

Following PSM, the multivariate Cox regression modeling found older age (hazard ratio $[\mathrm{HR}]=1.76$, 95\% confidence interval $[\mathrm{CI}]=1.51-2.06)$, more comorbidities $(\mathrm{HR}=1.46,95 \% \mathrm{CI}=1.25-1.70), \mathrm{EOE}(\mathrm{HR}=1.25$, $95 \% \mathrm{CI}=1.06-1.48)$, CBI $(\mathrm{HR}=1.20,95 \% \mathrm{CI}=1.02-1.40)$, and larger tumors $(\mathrm{HR}=1.52,95 \% \mathrm{CI}=1.40-1.66)$ as negative prognosticators of survival. Brachytherapy was a positive prognosticator of survival $(\mathrm{HR}=0.45,95 \%$ $\mathrm{CI}=0.40-0.51)$ (Table 8).

\section{Discussion}

In this large retrospective review, patients selected for brachytherapy had improved survival compared to enucleation in all size cohorts. This result persisted after propensity score matching. Given that the COMS medium trial was a prospective randomized trial showing equivalence of brachytherapy and enucleation for medium sized tumors, the superior OS for brachytherapy seen in our study was very likely due to selection bias in the data, as the patients who underwent enucleation were more like- ly to be uninsured, with a lack of financial resources, and having tumors too large to be amendable to EPBT [3]. Patients with large tumors selected for EPBT were likely to have smaller tumors on average than those who received enucleation. Since randomized evidence in large size tumors is lacking, our data can help to provide evidence for clinicians that brachytherapy is a reasonable treatment option for selected patients with large size tumors. EOE and CBI were significantly higher in the enucleation cohort and were likely to bias the clinician against treating with brachytherapy.

Mortality from ocular melanoma was initially believed by some to be from metastatic seeding at the time of enucleation, which was known as the "Zimmerman hypothesis" [17]. The COMS trial involving large size OM included patients with tumor height $>10 \mathrm{~mm}$ or diameter $>16 \mathrm{~mm}$ randomized to either external beam radiation (20 Gy in 5 fractions) before enucleation versus enucleation alone [18]. The concept for this approach was that pre-enucleation radiation could sterilize the area surrounding the tumor bed, decreasing the chance of metastatic seeding from the enucleation procedure. No significant difference was demonstrated for all-cause mortality or distant metastases at 5-year and 10-year follow-up. The findings disproved Zimmerman's hypothesis, suggesting that metastatic spread occurred prior to enucleation or other local treatment.

Recent studies use a 12-gene expression profiling (GEP) assay to classify the aggressiveness of OM tumors [19]. They are divided into $1 \mathrm{~A}, 1 \mathrm{~B}$, and 2 classes, with associated 5 -year metastatic risk of $2 \%, 21 \%$, and $72 \%$, respectively. In the highest risk group, class 2 , OM tumors are associated with larger tumor dimensions as well as GEP. Other high-risk features, such as CBI and EOE are associated with cytogenetic abnormalities [20]. Therefore, modern genomics has confirmed that the majority, if not all, of patients who undergo local therapy and then develop clinically evident metastases at a delayed time to have non-radiographically detectable micrometastases present at the time of diagnosis. Thus, which procedure is chosen for local control has only a marginal effect on survival outcomes. The COMS natural history study also 
A

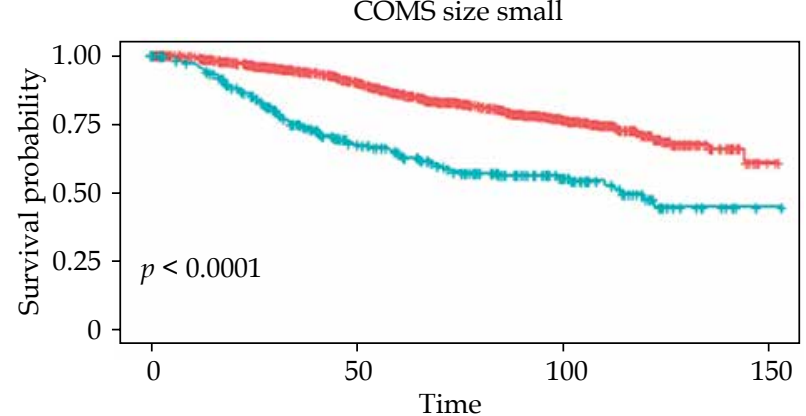

Number at risk

\begin{tabular}{|c|c|c|}
\hline$\underset{\mathbb{J}}{\mathbb{J}}$ therapy 1,183 & 724 & 268 \\
\hline $\begin{array}{l}\text { Enucle- } \\
\text { की }\end{array} 31$ & 147 & 54 \\
\hline
\end{tabular}

+ Brachytherapy $\quad+$ Enucleation

COMS size medium


+ Brachytherapy + Enucleation

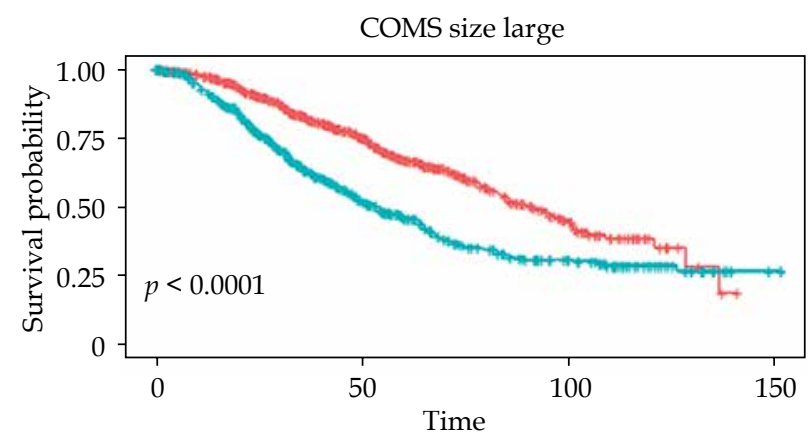

Number at risk

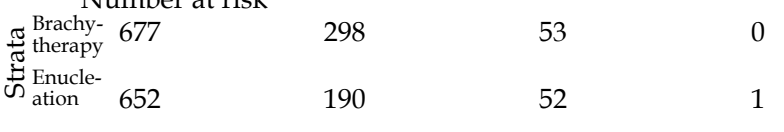

+ Brachytherapy + Enucleation
B



Number at risk

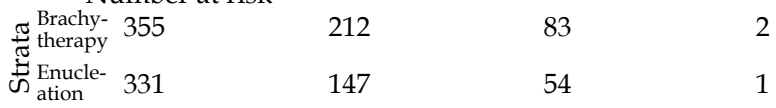

+ Brachytherapy $\quad+$ Enucleation
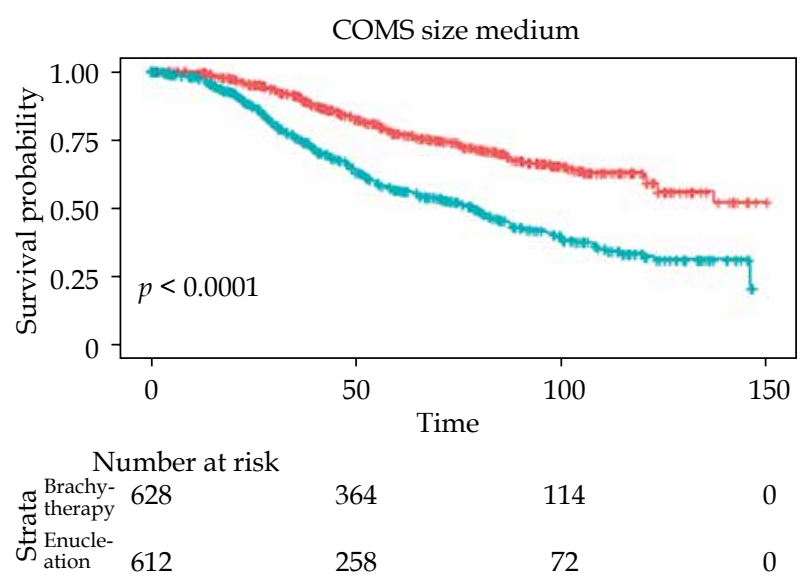

+ Brachytherapy $\quad+$ Enucleation

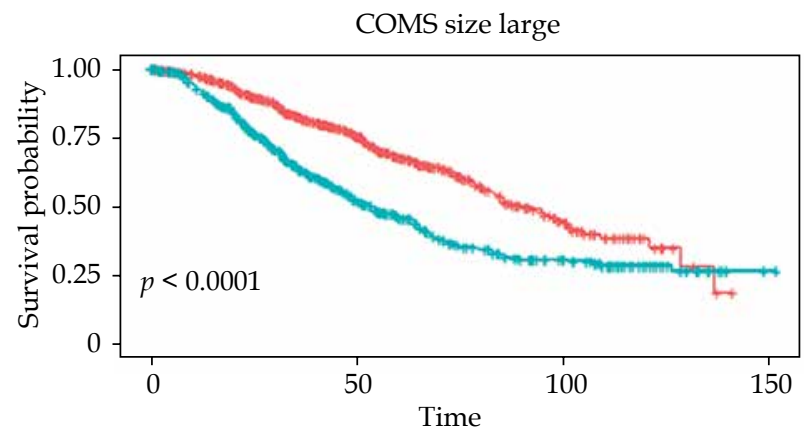

Number at risk

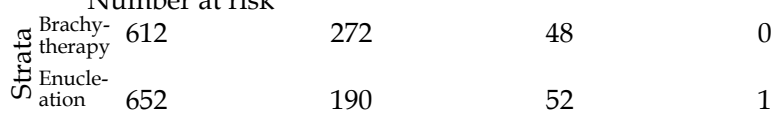

+ Brachytherapy + Enucleation

Fig. 2. Kaplan-Meier overall survival curves A) for all patients, B) after propensity score matching

confirmed this theory [21]. As such, EPBT may be a preferable treatment for these tumors since it allows for globe and vision preservation.

EPBT is an established treatment for small and medium sized tumors as discussed above. Retrospective studies and prospective case series regarding EPBT for large size tumors have demonstrated low rates of local re- currence, $6-9 \%$ at 5 years, which is comparable to COMS medium trial with $10.3 \%$ at 5 years (Table 9) $[3,4,7,8,22]$. The rates of metastasis mortality, $30-35 \%$ at 5 years, are comparable to COMS large trial with $28 \%$ at 5 years. Overall survival, $62 \%$ at 5 years in a retrospective paper, is in between COMS medium, $81 \%$ at 5 years, and COMS large trial, $57 \%$ at 5 years [8]. The 5 -yr OS of $67.9 \%$ for 
Table 5. Demographic data comparison after PSM

\begin{tabular}{|c|c|c|c|c|c|}
\hline$N=3,190$ & $\begin{array}{c}\text { Brachytherapy } \\
\text { alone } \\
(n=1,595)\end{array}$ & $\%$ & $\begin{array}{c}\text { Enucleation } \\
\text { alone } \\
(n=1,595)\end{array}$ & $\%$ & $p$-value \\
\hline Age ( $\geq 60$ years) & 892 & 56 & 871 & 55 & 0.455 \\
\hline Sex (male) & 897 & 56 & 910 & 57 & 0.642 \\
\hline Dx after 2010 & 691 & 43 & 645 & 40 & 0.099 \\
\hline Academic center & 1,046 & 66 & 1,076 & 68 & 0.260 \\
\hline White race & 1,503 & 94 & 1,503 & 94 & 1.000 \\
\hline Uninsured & 89 & 6 & 113 & 7 & 0.081 \\
\hline Private & 746 & 57 & 709 & 45 & 0.188 \\
\hline Government & 710 & 45 & 716 & 45 & 0.831 \\
\hline High school dropout > 21\% & 211 & 13 & 235 & 15 & 0.220 \\
\hline Median income $<\$ 38,000$ & 266 & 17 & 283 & 18 & 0.425 \\
\hline Population < 250,000 & 581 & 36 & 592 & 37 & 0.686 \\
\hline Distance to treatment $>60 \mathrm{mi}$ & 706 & 44 & 691 & 43 & 0.592 \\
\hline Charlson/Deyo score $\geq 1$ & 222 & 14 & 221 & 14 & 0.959 \\
\hline Extraocular extension & 156 & 10 & 237 & 15 & $<0.001^{\star}$ \\
\hline Choroid & 1,372 & 86 & 1,352 & 85 & 0.316 \\
\hline Ciliary & 223 & 14 & 243 & 15 & 0.316 \\
\hline COMS small & 355 & 22 & 331 & 21 & 0.301 \\
\hline COMS medium & 628 & 39 & 612 & 38 & 0.561 \\
\hline COMS large & 612 & 38 & 652 & 41 & 0.148 \\
\hline CD score 0 & 1,373 & 86 & 1,374 & 86 & \\
\hline 1 & 173 & 11 & 187 & 12 & \\
\hline 2 & 39 & 2 & 23 & 1 & \\
\hline 3 & 10 & 1 & 11 & 1 & \\
\hline
\end{tabular}

Table 6. 5-year overall survival (OS) for all patients after propensity score matching

\begin{tabular}{lccccc} 
Treatment & $n$ patients & Mean follow-up (months) & Median follow-up (months) & Range & 5-year OS \\
\hline Brachytherapy & 1,595 & 59 & 53 & $0-152$ & $75.8 \%$ \\
\hline Enucleation & 1,595 & 48 & 38 & $0-153$ & $54.0 \%$ \\
\hline Total & 3,190 & - & - & - & $p<0.001$
\end{tabular}

Table 7. 5-year overall survival (OS) for patients divided by COMS size after propensity score matching COMS size Brachytherapy cohort $\quad$ Enucleation cohort Log rank

\begin{tabular}{lccccc}
\cline { 2 - 4 } & $n$ patients & 5 -year OS & n patients & 5-year OS & \\
\hline Small & 355 & $86.5 \%$ & 331 & $64.1 \%$ & $p<0.001$ \\
\hline Medium & 628 & $77.1 \%$ & 612 & $56.6 \%$ & $p<0.001$ \\
\hline Large & 612 & $67.9 \%$ & 652 & $45.7 \%$ & $p<0.001$
\end{tabular}


Table 8. Cox proportional hazards multivariate model after propensity score matching

\begin{tabular}{|c|c|c|c|c|}
\hline \multirow[t]{2}{*}{ Parameter } & \multirow[t]{2}{*}{$H R$} & \multicolumn{2}{|c|}{$95.0 \% \mathrm{Cl}$} & \multirow[t]{2}{*}{$p$-value } \\
\hline & & Lower & Upper & \\
\hline Age & 1.764 & 1.512 & 2.058 & $<0.001$ \\
\hline Charlson/Deyo & 1.459 & 1.251 & 1.702 & $<0.001$ \\
\hline Extraocular extension & 1.254 & 1.064 & 1.478 & 0.007 \\
\hline Larger tumor size & 1.523 & 1.401 & 1.655 & $<0.001$ \\
\hline Brachytherapy & 0.451 & 0.399 & 0.509 & $<0.001$ \\
\hline Ciliary body invasion & 1.196 & 1.023 & 1.4 & 0.025 \\
\hline
\end{tabular}

$H R$ - hazard ratio, Cl-confidence interval

Table 9. Comparison of papers regarding eye plaque brachytherapy used to treat large size tumors to COMS trials

\begin{tabular}{|c|c|c|c|c|c|c|}
\hline Trial & Study & $n$ & Time & $\begin{array}{l}\text { Local } \\
\text { recurrence }\end{array}$ & $\begin{array}{c}\text { Metastasis } \\
\text { mortality }\end{array}$ & Overall survival \\
\hline \multirow[t]{2}{*}{ COMS medium } & \multirow{2}{*}{$\begin{array}{l}{ }^{125} \text { I plaque vs. } \\
\text { enucleation }\end{array}$} & \multirow[t]{2}{*}{1,317} & 5 years & $10.3 \%$ & $9 \%$ & $81 \%$ \\
\hline & & & 12 years & - & $21 \%$ & $57 \%$ \\
\hline \multirow[t]{2}{*}{ COMS large } & \multirow{2}{*}{$\begin{array}{c}\text { EBRT before } \\
\text { enucleation vs. } \\
\text { enucleation alone }\end{array}$} & \multirow[t]{2}{*}{1,003} & 5 years & N/A & $28 \%$ & $57 \%$ \\
\hline & & & 10 years & $\mathrm{N} / \mathrm{A}$ & $60 \%$ & $40 \%$ \\
\hline \multirow[t]{2}{*}{ Shields et al. } & \multirow{2}{*}{ 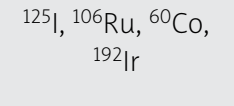 } & \multirow[t]{2}{*}{354} & 5 years & $9 \%$ & $30 \%$ & - \\
\hline & & & 10 years & $13 \%$ & $55 \%$ & - \\
\hline Puusaari et al. & 125 & 121 & 5 years & $6 \%$ & $35 \%$ & $62 \%$ \\
\hline
\end{tabular}

large tumors after PSM in our data is comparable to the 5-yr OS found in the retrospective paper.

The retrospective nature of this analysis is recognized as a limitation. While the NCDB only represents a percentage of patients treated in the United States, it has benefits over surveillance, epidemiology, and end results program (SEER) database, since it includes important clinical factors, such as tumor depth and size as well as socioeconomic factors [23]. However, the NCDB has additional limitations, including a lack of information regarding the gene expression profile of the tumors. There is a lack of information regarding local control, metastasis mortality, dose for brachytherapy, and toxicity. Potential adverse events from EPBT include scleral necrosis, strabismus, cataract, glaucoma, and retinopathies [24]. While PSM was used to reduce selection bias and potential clinical or demographic cofounders, even this analysis cannot completely eliminate confounding biases. Further prospective studies are warranted to determine criteria to select patients with large size tumors for brachytherapy to increase eye preservation rates.

\section{Conclusions}

Ocular melanoma is a rare disease with worse survival than other melanomas. Tumor genetics, size, and early micrometastatic spread are the most important indicators for survival. Therefore, local treatments other than enu- cleation, such as eye plaque brachytherapy, should be safe to use to preserve a patient's vision and aesthetics. To our knowledge, this study is the largest study of eye plaque brachytherapy versus enucleation, and our findings suggest that EPBT is a reasonable treatment option for all size ocular melanoma tumors, including large size tumors.

\section{Disclosure}

The authors report no conflict of interest.

\section{References}

1. Krantz BA, Dave N, Komatsubara KM et al. Uveal melanoma: epidemiology, etiology, and treatment of primary disease. Clin Ophthalmol 2017; 11: 279-289.

2. Egan KM, Seddon JM, Glynn RJ et al. Epidemiologic aspects of uveal melanoma. Surv Ophthalmol 1988; 32: 239-251.

3. Margo CE. The COMS randomized trial of iodine 125 brachytherapy for choroidal melanoma V. Twelve-year mortality rates and prognostic factors: COMS Report No. 28. Arch Ophthalmol 2006; 124: 1684-1693.

4. Diener-West M, Earle JD, Fine SL et al. The COMS randomized trial of iodine 125 brachytherapy for choroidal melanoma, III: initial mortality findings. COMS Report No. 18. Arch Ophthalmol 2001; vol. 119: 969-982.

5. DiMaio D et al. NCCN Guidelines Version 1.2019 Uveal Melanoma, National Comprehensive Cancer Network, 2019. Available: https://www.nccn.org/professionals/physician_ gls/pdf/uveal.pdf (accessed: 12 Jun 2019). 
6. Simpson ER, Gallie B, Laperrierre N et al. The American Brachytherapy Society consensus guidelines for plaque brachytherapy of uveal melanoma and retinoblastoma. Brachytherapy 2014; 13: 1-14.

7. Shields CL, Naseripour M, Cater J et al. Plaque radiotherapy for large posterior uveal melanomas ( $\geq 8$-mm thick) in 354 consecutive patients. Ophthalmology 2002; 109: 1838-1849.

8. Puusaari I, Heikkonen J, Summanen $\mathrm{P}$ et al. Iodine brachytherapy as an alternative to enucleation for large uveal melanomas. Ophthalmology 2003; 110: 2223-2234.

9. Tann AW, Teh BS, Scarboro SB et al. Early outcomes of uveal melanoma treated with intraoperative ultrasound guided brachytherapy using custom built plaques. Pract Radiat Oncol 2017; 7: e275-e282.

10. Lewis GD, Haque W, Butler EB et al. Survival outcomes and patterns of management for anal adenocarcinoma. Ann Surg Oncol 2019; 26: 1351-1357.

11. Lewis GD, Dalwadi SM, Farach A et al. The role of adjuvant radiotherapy in the treatment of pleural mesothelioma. Ann Surg Oncol 2019; 26: 1879-1885.

12. Haque $W$, Verma V, Lewis GD et al. Utilization of radiotherapy and stereotactic body radiation therapy for renal cell cancer in the USA. Future Oncol 2018; 14: 819-827.

13. Haque $W$, Lewis GD, Verma $V$ et al. The role of adjuvant chemotherapy in locally advanced bladder cancer. Acta Oncol 2018; 57: 509-515.

14. Charlson ME, Pompei P, Ales KL et al. A new method of classifying prognostic comorbidity in longitudinal studies: Development and validation. J Chronic Dis 1987; 40: 373-383.

15. Randolph JJ, Falbe K, Kureethara Manuel A et al. A Step-bystep guide to propensity score matching in R. Pract Assessment Res Eval 2014; 19: 1-6.

16. McLaughlin CC, Wu XC, Jemal A et al. Incidence of noncutaneous melanomas in the U.S. Cancer 2005; 103: 1000-1007.

17. Shields JA, Shields CL. Management of posterior uveal melanoma: past, present, and future: the 2014 Charles L. Schepens lecture. Ophthalmology 2015; 122: 414-428.

18. Margo CE. The Collaborative Ocular Melanoma Study (COMS) randomized trial of pre-enucleation radiation of large choroidal melanoma: IV. Ten-year mortality findings and prognostic factors. COMS report number 24. Am J Ophthalmol 2004; 138: 936-951.

19. Walter SD, Chao DL, Feuer W et al. Prognostic implications of tumor diameter in association with gene expression profile for uveal melanoma. JAMA Ophthalmol 2016; 134: 734-740.

20. Shields CL, Say EAT, Hasanreisoglu M et al. Cytogenetic abnormalities in uveal melanoma based on tumor features and size in 1059 patients: the 2016 W. Richard Green lecture. Ophthalmology 2017; 124: 609-618.

21. Straatsma B, Diener-West M, Caldwell R et al. Mortality after deferral of treatment or no treatment for choroidal melanoma. Indian J Ophthalmol 2018; 66: 1395-1400.

22. Margo CE. The Collaborative Ocular Melanoma Study (COMS) randomized trial of pre-enucleation radiation of large choroidal melanoma ii: initial mortality findings COMS Report No. 10. Am J Ophthalmol 1998; 9394: 779-796.

23. Rao YJ, Sein J, Badiyan S et al. Patterns of care and survival outcomes after treatment for uveal melanoma in the postCOMS era (2004-2013): a surveillance, epidemiology, and end results analysis. J Contemp Brachytherapy 2017; 9: 453-465.

24. Peddada KV, Sangani R, Menon H et al. Complications and adverse events of plaque brachytherapy for ocular melanoma. J Contemp Brachytherapy 2019; 11: 392-397. 